\title{
Association between organizational inequity and incidence of psychiatric disorders in female employees
}

\author{
M. KIVIMÄKI, ${ }^{1}$ M. ELOVAINIO, J. VAHTERA, M. VIRTANEN \\ AND S. A. STANSFELD \\ From the University of Helsinki, Department of Psychology, Finnish Institute of Occupational Health, \\ Helsinki and Turku, and Research and Development Centre for Health and Welfare, Helsinki, Finland; \\ and Department of Psychiatry, Queen Mary College, University of London
}

\begin{abstract}
Background. Prior research on work-related factors in the aetiology of psychiatric disorders has been concentrated on job control, job demands and social support. To broaden the view to managerial procedures, we examined whether the extent to which employees are treated with equity in the workplace predicts their mental health. Organizational equity refers to decision-making procedures, which are consistently applied, open, correctable and include input from affected parties (procedural justice). It also refers to respectful and considerate treatment of individuals by supervisors (relational justice).
\end{abstract}

Method. A cohort of 1786 female hospital employees with no psychiatric disorder at baseline responded to a questionnaire on organizational equity. From the responses, both an individual score and a work unit mean score were assigned to each participant. The outcome was new reports of doctor-diagnosed psychiatric disorders during the 2-year follow-up. Odds ratios and $95 \%$ confidence intervals were corrected for clustering of the data.

Results. After adjustment for age and salary, odds ratio of new psychiatric disorders for self-reported low procedural justice was $1.9(95 \%$ CI $1 \cdot 1$ to $3 \cdot 2)$. Corresponding odds ratio for low procedural justice, as assessed with work unit mean scores, was 1.7 (95\% CI 1.5 to 2.0$)$. These associations remained statistically significant after additional adjustment for mental distress at baseline and job control, job demands and social support. Relational justice did not predict psychiatric disorders.

Conclusion. Research on organizational equity provides new information about potential workrelated determinants of mental health. Low procedural justice seems to be an independent risk factor for psychiatric disorders in female employees.

\section{INTRODUCTION}

Studies on social work environment and psychiatric disorders have typically focused on job control (i.e. decision authority and skill discretion), job demands and social support (Stansfeld et al. 1997, 1999; Wall et al. 1997). However, organizational equity may also play an important

\footnotetext{
1 Address for correspondence: Professor Mika Kivimäki, University of Helsinki, Department of Psychology, Division of Applied Psychology, PO Box 9, FIN-00014 University of Helsinki, Finland.
}

role in the well-being of employees (Elovainio et al. 2001, 2002).

The term 'organizational equity' (or 'organizational justice') refers to the extent to which employees are treated with justice at their workplace (Moorman, 1991; Cropanzano et al. 2001). Organizational equity involves a procedural component and a relational component. The former indicates whether decision-making procedures include input from affected parties, are consistently applied, suppress bias, are accurate, 


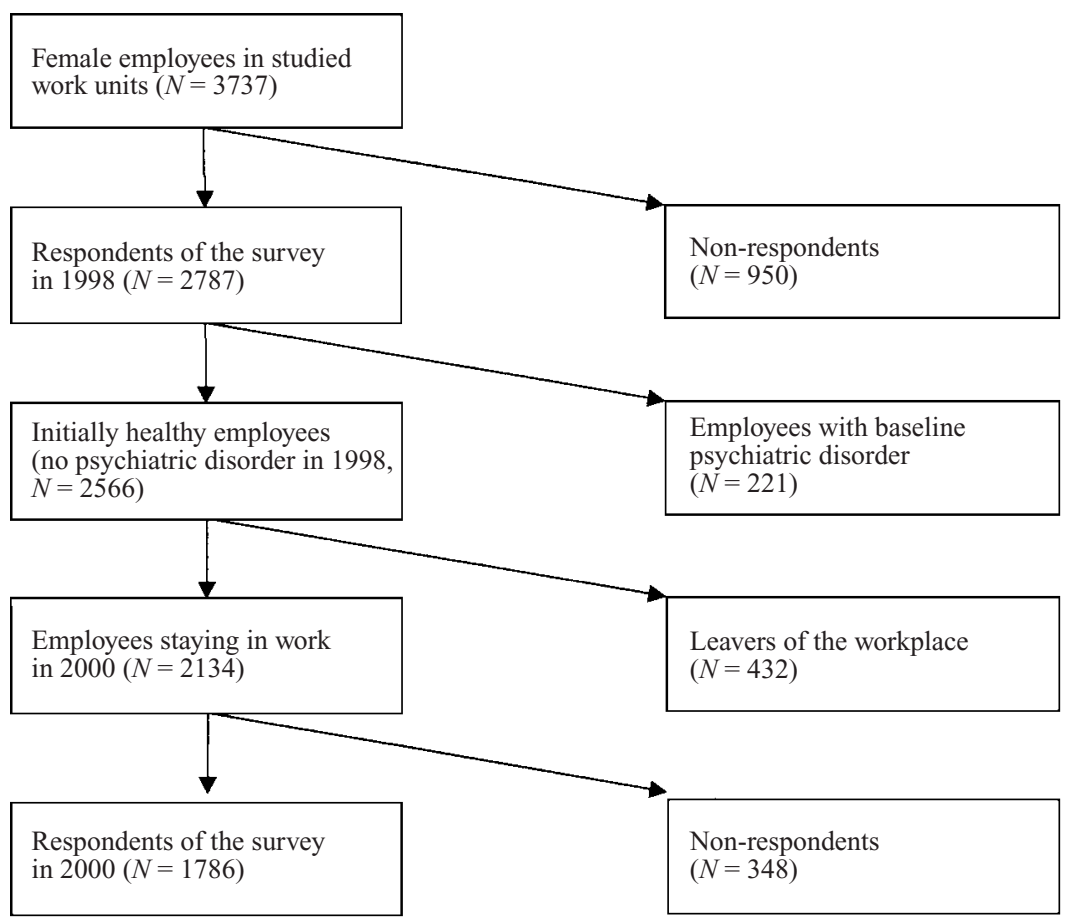

FIG. 1. Flow chart of the sample selection procedure.

are correctable and are ethical (called procedural justice). The latter element refers to treating individuals with politeness and consideration by supervisors (called relational justice).

Organizational inequity has been associated with job dissatisfaction, retaliation and lower work commitment (Moorman, 1991; Dailey \& Kirk, 1992; Shapiro \& Brett, 1993). Previous research also suggests that low perceived equity may be related to factors that influence susceptibility to illness, such as elevated serum lipids and negative feelings (Shapiro \& Brett, 1993; Richards et al. 2000; Elovainio et al. 2001). Our own recent work from a cross-sectional study of Finnish hospital personnel has shown low procedural justice to be associated with increased risk of mental distress (as assessed by the General Health Questionnaire), sickness absence and poor self-rated health status (Elovainio et al. 2002). Relational justice was less consistently associated with these outcomes.

Psychiatric disorders are among the most common causes of disability retirement in workers and a source of personal suffering (Pattani et al. 2001). To date, no prior research has been published on the association between organizational equity and psychiatric disorders. However, such an association can be expected considering the observed links between organizational equity, negative feelings and mental distress (Elovainio et al. 2000, 2001).

In this study, we have investigated organizational inequity as a risk factor for doctor-diagnosed psychiatric disorders using prospective data and taking into account baseline mental distress in the design and analysis as well as relevant psychological and social factors.

\section{METHOD}

\section{Study sample}

Fig. 1 summarizes the sample selection procedure. All 10 hospitals in two of the 23 health districts in Finland volunteered to participate in a project 'Work and health in Finnish hospital personnel' coordinated by the Finnish Institute of Occupational Health (Kivimäki et al. 2000a). Based on employers' registers of the hospitals, there were 162 single-location work units with more than 10 employees. In 1998, all female 
employees working in these work units were asked to respond to a questionnaire on organizational equity, psychiatric disorders and other variables. In 2000 , a follow-up survey was sent to those respondents of the baseline survey who remained in the service of the hospitals and reported no doctor-diagnosed psychiatric disorder at baseline. Response rates for the first and second surveys were $75 \%$ and $84 \%$, respectively. The final cohort comprised 1786 women of which $58(3 \%)$ were doctors, $1363(76 \%)$ nurses, $149(8 \%)$ administrative staff, $81(5 \%)$ X-ray and laboratory staff, and $135(8 \%)$ maintenance, cleaning and other staff (Table 1).

The final cohort did not differ from the eligible 3737 female employees in terms of mean age $(43 \cdot 5$ $v .43 .0$ years in the participants and the eligible population, respectively) or mean monthly salary (FIM $10979 v$. 10923). The level of organizational equity in the final cohort corresponded well with that among the respondents of the first survey. The scores of procedural justice and relational justice were 2.71 and 3.59 in the first group and 2.73 and 3.60 in the latter group. Thus, bias due to selection procedures or sample attrition seems unlikely in the present study.

\section{Measurements}

Components of organizational equity were measured by a procedural justice scale and a relational justice scale (Moorman, 1991). The former measure (seven items, Cronbach's alpha $(\alpha)=0.80)$ requests the degree to which the respondent considers the procedures used at the workplace to be designed to collect accurate information necessary for making decisions, to provide opportunities to appeal or challenge the decision, to generate standards so that decisions can be made with consistency, and to hear the concerns of all those affected by the decision. Relational justice scale (six items, $\alpha=0.90$ ) requests whether the respondent thinks that his/her supervisor is able to suppress personal biases, treats subordinates with kindness and consideration, and takes steps to deal with subordinates in a truthful manner. In both scales, responses are given along a 5-point scale from $1=$ strongly disagree to $5=$ strongly agree. For each participant, we derived an individual score and a work unit mean score (the work unit mean score applied to all members of the work unit) from the responses to the baseline survey.
Respondents were asked to indicate presence of diseases using a checklist of 14 common chronic diseases (Vahtera et al. 1997; Kivimäki et al. 2000a). Doctor-diagnosed psychiatric disorder depended on whether the subject reported that a general practitioner or a hospital doctor had confirmed either 'clinical depression' or 'some other mental illness'. Doctor-diagnosed psychiatric disorder was assessed in the baseline survey and follow-up survey.

Covariates were measured at baseline. Demographic factors included age and salary. Mental distress was assessed using the 12-item version of the General Health Questionnaire (GHQ-12) (Goldberg \& Williams, 1998). Individuals scoring $\geqslant 4$ are estimated to have mental distress according to studies validating GHQ-12 against standardized psychiatric interviews (Goldberg \& Williams, 1988), and this threshold was applied in the present study to identify people with mental distress at baseline. Scales assessing social factors were decision authority (three items, range $=1-5, \alpha=0 \cdot 81$ ) and skill discretion (six items, range $=1-5, \alpha=0 \cdot 81)$ (Karasek, 1985), as dimensions of job control, job demands (five items, range $=1-5, \alpha=0.83$ ) (Harris, 1985) and social support (six items, range $=0-30, \alpha=0 \cdot 82$ ) (Sarason et al. 1987). These well-validated scales have successfully predicted health in prior prospective cohort studies (e.g. Bosma et al. 1997; Kivimäki et al. 1997, 2000 a, b; Ferrie et al. 1998; Vahtera et al. 2000). Life style factors were measured by standard methods: smoking status (current smoker $v$. not), alcohol consumption (high consumption $>190 \mathrm{~g}$ of absolute alcohol per average week) (Kaprio et al. 1987) and sedentary life-style (less than half an hour of fast walking per week) (Kivimäki et al. 1998; Kujala et al. 1998). Biological factors included obesity (body mass index $>30 \mathrm{~kg} / \mathrm{m}^{2}$ ), reported doctordiagnosed hypertension and high cholesterol.

\section{Statistical analysis}

The data were likely to be clustered. Employees who had chosen the same work unit or workplace might be expected to have something in common. Responding to questionnaire could be affected by other employees in the same work unit or workplace. Traditional regression modelling is based on the assumption of independent observations and use of such modelling strategy for clustered data could lead to inaccurate estimation. For 
Table 1. Characteristics of respondents at baseline

\begin{tabular}{|c|c|c|}
\hline Baseline characteristic & Mean (S.D.) & $N(\%)$ \\
\hline \multicolumn{3}{|l|}{ Demographic characteristics } \\
\hline Age (years) & $43 \cdot 5(8 \cdot 1)$ & \\
\hline Salary (1000 FIM per month) & $11 \cdot 0(2 \cdot 2)$ & \\
\hline \multicolumn{3}{|l|}{$\begin{array}{l}\text { Subclinical or undiagnosed } \\
\text { psychiatric disorders }\end{array}$} \\
\hline Mental distress & & $353(20)$ \\
\hline \multicolumn{3}{|l|}{ Psychosocial factors } \\
\hline Decision authority & $3 \cdot 51(0 \cdot 87)$ & \\
\hline Skill discretion & $3.87(0.57)$ & \\
\hline Job demands & $3.61(0.85)$ & \\
\hline Social support & $12 \cdot 7(5 \cdot 3)$ & \\
\hline \multicolumn{3}{|l|}{ Life style factors } \\
\hline Current smoking & & $202(11)$ \\
\hline Heavy alcohol consumption & & $132(7)$ \\
\hline Sedentary life style & & $306(17)$ \\
\hline \multicolumn{3}{|l|}{ Biological factors } \\
\hline Obesity & & $142(8)$ \\
\hline Hypertension & & $124(7)$ \\
\hline High cholesterol & & $148(9)$ \\
\hline \multicolumn{3}{|l|}{ Organizational equity } \\
\hline Procedural justice & $2 \cdot 72(0 \cdot 71)$ & \\
\hline Relational justice & $3.59(0.95)$ & \\
\hline
\end{tabular}

this reason, we used multilevel logistic regression analysis to estimate the strength of the association between organizational equity and incidence of psychiatric disorders. Employees represented the individual level and work units $(N=162)$ or hospitals $(N=10)$ the cluster level. The number of participants in the work units varied between 1 and 35 (mean 11.0) and that in hospitals between 39 and 753 (mean 178.6). We used generalized estimation equations with an exchangeable correlation structure corresponding to a random effect to correct for work unit in relation to individual scores of organizational equity. Regarding work unit mean scores, analyses were corrected for hospital level.

Components of organizational equity were standardized and treated as continuous variables. The odds ratios for new doctor-diagnosed psychiatric disorders for procedural and relational justice at high and low levels were calculated. The cut-points for high and low justice were set at +1 standard deviation (s.D.) and -1 s.D., respectively. We adjusted odds ratios and their $95 \%$ CI for demographics (age and salary), mental distress (GHQ-caseness), social factors (decision authority, skill discretion, job demands, social support), life style factors (smoking, alcohol consumption, sedentary life style) and biological factors (obesity, hypertension, high cholesterol). Mental distress, and biological and life style variables were dichotomous. Social factors were treated as continuous variables and standardized.

For all analyses, we used the SAS 8.12 program package.

\section{RESULTS}

Baseline characteristics of the participants are shown in Table 1. Mean age was 44 (range 20-63) years. One in five had mental distress at baseline, one in six had a sedentary life style, and about one in ten was a current smoker, heavy alcohol consumer, obese, hypertensive and had a high cholesterol level. Eighty-four initially healthy participants reported a new doctor-diagnosed psychiatric disorder in the follow-up survey (incidence $4 \cdot 7 \%$ ).

Table 2. Odds ratios (95\% confidence intervals) of new doctor-diagnosed psychiatric disorders for levels of procedural justice

\begin{tabular}{|c|c|c|c|}
\hline \multirow[b]{2}{*}{ Characteristic* } & \multirow[b]{2}{*}{ Unadjusted } & \multicolumn{2}{|c|}{ Adjusted for } \\
\hline & & Age and salary & Mental distress at baseline $\dagger$ \\
\hline \multicolumn{4}{|c|}{ Procedural justice (individual score) } \\
\hline High & 1.00 & $1 \cdot 00$ & $1 \cdot 00$ \\
\hline Low & $1 \cdot 91(1 \cdot 14$ to $3 \cdot 19)$ & $1 \cdot 90(1 \cdot 14$ to $3 \cdot 17)$ & $1.73(1.02$ to 2.93$)$ \\
\hline \multicolumn{4}{|c|}{ Procedural justice (work unit mean score } \\
\hline High & 1.00 & $1 \cdot 00$ & $1 \cdot 00$ \\
\hline Low & $1 \cdot 73(1.48$ to $2 \cdot 03)$ & $1.77(1.52$ to $2 \cdot 07)$ & $1.65(1.36$ to $2 \cdot 00)$ \\
\hline
\end{tabular}

\footnotetext{
* High and low levels of procedural justice refer to +1 S.D. and -1 S.D., respectively.

$\uparrow$ The 12-item General Health Questionnaire score four or more.

\$ The work unit mean score of procedural justice applied to all members of the work unit.
} 
Table 3. Odds ratios (95\% confidence intervals) of new doctor-diagnosed psychiatric disorders for levels of relational justice

\begin{tabular}{|c|c|c|c|}
\hline \multirow[b]{2}{*}{ Characteristic* } & \multirow[b]{2}{*}{ Unadjusted } & \multicolumn{2}{|c|}{ Adjusted for } \\
\hline & & Age and salary & Mental distress at baseline $\dagger$ \\
\hline \multicolumn{4}{|c|}{ Relational justice (individual score) } \\
\hline High & $1 \cdot 00$ & $1 \cdot 00$ & $1 \cdot 00$ \\
\hline Low & $1 \cdot 36(0 \cdot 84$ to $2 \cdot 19)$ & $1 \cdot 34(0 \cdot 83$ to $2 \cdot 15)$ & $1.24(0.77$ to 1.99$)$ \\
\hline \multicolumn{4}{|c|}{ Relational justice (work unit mean score:) } \\
\hline High & 1.00 & $1 \cdot 00$ & $1 \cdot 00$ \\
\hline Low & $1.29(0.71$ to 2.34$)$ & $1.29(0.70$ to 2.39$)$ & $1.20(0.62$ to 2.35$)$ \\
\hline
\end{tabular}

$*$ High and low levels of relational justice refer to +1 s.D. and -1 s.D., respectively.

$\dagger$ The 12-item General Health Questionnaire score four or more.

\$ The work unit mean score of relational justice applied to all members of the work unit.

Table 4. Effect of adjustments on the association between procedural justice and new doctordiagnosed psychiatric disorders (figures are odds ratios (95\% confidence intervals))

\begin{tabular}{|c|c|c|c|}
\hline \multirow[b]{2}{*}{ Characteristic* } & \multicolumn{3}{|c|}{ Adjustment for age, salary and } \\
\hline & Social factors $\dagger$ & Life style factors: & Biological factors $\S$ \\
\hline \multicolumn{4}{|c|}{ Procedural justice (individual score) } \\
\hline High & $1 \cdot 00$ & $1 \cdot 00$ & 1.00 \\
\hline Low & $2 \cdot 04(1 \cdot 18$ to $3 \cdot 51)$ & $1 \cdot 87(1.08$ to $3 \cdot 23)$ & $1.64(0.95$ to $2 \cdot 81)$ \\
\hline \multicolumn{4}{|c|}{ Procedural justice (work unit mean score } \\
\hline High & 1.00 & 1.00 & 1.00 \\
\hline Low & $1.75(1.47$ to 2.09$)$ & $1.59(1.37$ to 1.86$)$ & $1 \cdot 77(1.51$ to $2 \cdot 07)$ \\
\hline
\end{tabular}

\footnotetext{
* High and low levels of procedural justice refer to +1 S.D. and -1 s.D., respectively.

$\dagger$ Decision authority, skill discretion, job demands, and social support.

* Smoking, alcohol consumption, and sedentary life style.

$\S$ Obesity, hypertension, and high cholesterol.

- The work unit mean score of procedural justice applied to all members of the work unit.
}

\section{Associations of procedural and relational justice with incident psychiatric disorder}

Table 2 presents results on the associations between procedural justice and incidence of psychiatric disorders. Age- and salary-adjusted odds ratios of new psychiatric disorder for low procedural justice, as assessed with individual scores and work unit mean scores, were 1.9 and 1.7 , respectively. Both odds ratios remained statistically significant after an additional adjustment for mental distress at baseline.

Table 3 shows that the association between relational justice and incidence of psychiatric disorders was not significant in unadjusted or adjusted regression models.

\section{Contribution of social, life style and biological factors to the association}

Table 4 shows how adjustment for social, life style and biological factors affected the association between procedural justice and incidence of psychiatric disorders. Compared to age- and salaryadjusted figures, additional adjustment for social factors slightly increased the odds ratio of selfreported procedural justice. Adjustment for biological factors slightly reduced the odds ratios of self-reported procedural justice and adjustment for life style factors slightly reduced the odds ratios of procedural justice, as assessed with work unit mean scores. Except in the former case, the association between procedural justice and psychiatric disorders remained statistically significant after adjustments for the 12 different covariates. This supports the hypothesis that procedural justice independently affects mental health.

\section{DISCUSSION}

This is apparently the first longitudinal study to show that the extent to which managerial 
procedures are fair in the workplace is associated with the risk of psychiatric disorders in employees. Our findings rely on longitudinal data of initially healthy participants and statistical analysis which takes into account of clustering. The results persist after adjustment for baseline mental distress. Use of work unit mean scores in the assessment of organizational equity decreased risk of reporting bias and conflation between exposure and outcome. The surveys achieved $75-84 \%$ response rates, which are satisfactory for studies of this kind (Nachmias \& Nachmias, 1981). Furthermore, comparison of participants and the eligible population show that sample attrition is an unlikely source of confounding in this investigation.

Several studies suggest that psychosocial work characteristics may be important determinants of mental ill health. For example, Stansfeld et al. found that low decision authority, high job demands and insufficient social support were associated with increased risk of psychiatric disorders (Stansfeld et al. 1999). A study of the UK National Health Service (NHS) work force reported that high work demands were associated with worse mental health (Wall et al. 1997). In the present study, adjustment for job control, job demands and social support did not attenuate the association between procedural justice and psychiatric disorders. This suggests that the contribution of fair decision-making procedures at workplace to mental health is not attributable to relations between other major psychosocial factors and psychiatric disorders.

We found that control for life style and biological health risk factors slightly attenuated the association between procedural justice and psychiatric disorders. Different types of risk factors tend to concentrate among the same people and prior research suggests interrelations between stressful work characteristics, smoking, heavy alcohol intake, obesity, high blood pressure, serum lipids and mental health problems (Shapiro \& Brett, 1993; Williams et al. 1998; Hemingway \& Marmot, 1999; Richards et al. 2000; Tanskanen et al. 2000).

Both procedural justice and relational justice deal with how supervisors relate to their employees. This relationship may be crucial for mental health. Research on depression in women suggests that life events in combination with humiliation and devaluation are more likely to lead to depression than life events alone (Brown et al. 1995). Humiliation of employees may well be a component of low relational justice. It is also possible that poor relations between supervisors and employees are a result of the supervisors being treated badly themselves by their superiors. Such a hierarchy of poor relations may be a reflection of a malfunctioning organization in which low procedural justice may be either a cause or a consequence.

In the present study, relational justice was not independently associated with incidence of psychiatric disorders. In accordance, prior research reports less consistent health effects for relational justice than for procedural justice (Elovainio et al. 2001). Impolite, inconsiderate supervision may not be a major contributing factor to psychiatric disorders at least when relational justice is not extremely low. In contrast, severe relational injustice, as indicated by workplace bullying, has been found to increase morbidity among the bullied (Kivimäki et al. 2000).

\section{Limitations of the study}

Measuring organizational equity with more objective indicators would be a step forward. This study assessed organizational equity and psychiatric disorders with self-reports. It is well known that common-method variance may artificially inflate associations in cross-sectional data, e.g. through negative and positive response sets. Because we measured incidence (i.e. change in mental health between the two surveys), an artificial inflation of associations would have occurred only if common-method variance had affected the second survey but not the first survey. We believe that this is a very unlikely alternative. Use of work unit mean scores further reduced risk of bias due to common-method variance.

Employees with doctor-diagnosed psychiatric disorders at baseline were excluded from the data in order to establish temporal order between constructs. However, all people with mental illness may not get diagnosed. To uncover this problem, models on the association between organizational equity and diagnosed psychiatric disorders were adjusted for baseline mental distress. In future research, a complementary use of psychiatric interview, both at baseline and follow-up, may further strengthen the validity of assessing incident psychiatric disorders. 
Further research is also needed to examine whether the present findings can be generalized to men and non-hospital settings.

\section{Practical implications}

Population-based interventions for improving mental health are difficult to achieve. However, the workplace may be a potentially feasible area for interventions for reducing risk of psychiatric disorders. The recommendations made in the Nuffield report for improving the mental health of NHS employees are in line with the present findings in the Finnish hospital staff (Williams et al. 1998). The report states that 'management style clearly affects health'. Recommended actions included improving 'two way communications' and developing 'a culture in which staff are valued', which is keeping with the powerful effects of effort/reward imbalance on mental health (Stansfeld et al. 1999). Our findings suggest that it may also be important to ensure that organizational policies, practices and procedures include input from affected parties, are consistently applied, suppress bias, and are accurate, correctable and ethical. Organizational equity may communicate status and value in a group, and for this reason people care about fair treatment by authorities. Efforts to follow such principles at workplaces are probably not in contrast to business efficiency.

\section{Conclusion}

This study provides new information about work-related determinants of psychiatric disorders. All the findings reported here suggest that procedural justice at the workplace may be a crucial and independent aspect of the social environment influencing mental ill health in working populations. The traditional focus on job control, job demands, and social support has been an important first step, but broadening the view to managerial procedures seems now to be important. Such a perspective may not only increase our understanding of the work-related risks but also suggests new priorities for strategies of promotion of mental health and wellbeing at workplaces.

Mika Kivimäki was supported by the Finnish Work Environment Fund (FWEF) (project 97316) and the Academy of Finland (projects 44968 and 77560). Marko Elovainio and Jussi Vahtera were supported by the Finnish Work Environment Fund (projects 96052 and 801639). The on-going project 'Work and health in Finnish hospital personnel' is additionally supported by the participating hospitals. We thank Dr Hans Helenius for his help in statistical analysis.

\section{REFERENCES}

Bosma, H., Marmot, M. G., Hemingway, H., Nicholson, A. C., Brunner, E. \& Stansfeld, S. A. (1997). Low job control and risk of coronary heart disease in the Whitehall II (prospective cohort) study. British Medical Journal 314, 558-564.

Brown, G. W., Harris, T. O. \& Hepworth, C. (1995). Loss, humiliation and entrapment among women developing depression: a patient and non-patient comparison. Psychological Medicine $\mathbf{2 5}$, $7-21$.

Cropanzano, R., Byrne, Z. S., Bobocel, D. R. \& Rupp, D. E. (2001) Moral virtues, fairness heuristics, social entities, and other denizens of organizational justice. Journal of Vocational Behavior $\mathbf{5 8}$, 164-209.

Dailey, R. C. \& Kirk, D. J. (1992). Distributive and procedural justice as antecedents of job dissatisfaction and intent to turnover. Human Relations 45, 305-317.

Elovainio, M., Kivimäki, M. \& Helkama, K. (2001). Organizational justice evaluations, job control, and occupational strain. Journal of Applied Psychology 86, 418-424.

Elovainio, M., Kivimäki, M. \& Vahtera, J. (2002). Organizational justice: evidence of a new psychosocial predictor of health. American Journal of Public Health 92, 105-108.

Ferrie, J. E., Shipley, M. J., Marmot, M. G., Stansfeld, S. A. \& Smith, G. D. (1998). An uncertain future: the health effects of threats to employment security in white-collar men and women. American Journal of Public Health 88, 1030-1036.

Goldberg, D. \& Williams, P. (1988). A User's Guide to the General Health Questionnaire. NFER-Nelson Publishing Co.: Windsor.

Harris, P. E. (1985). The nurse stress index. Work and Stress 3 335-345.

Hemingway, H. \& Marmot, M. (1999). Psychosocial factors in the aetiology and prognosis of coronary heart disease: systemic review of prospective cohort studies. British Medical Journal 319 , 1460-1467.

Kaprio, J., Koskenvuo, M., Langinvainio, H., Romanov, K., Sarna, S. \& Rose, R. J. (1987). Genetic influences on use and abuse of alcohol: a study of 5638 adult Finnish twin brothers. Alcoholism. Clinical and Experimental Research 11, 349-356.

Karasek, R. (1985). Job Content Questionnaire and User's Guide. Revision 1.1. Lowell, M. A., University of Massachusetts, Department of Work Environment: Massachusetts.

Kivimäki, M., Vahtera, J., Thomson, L., Griffiths, A., Cox, T. \& Pentti, J. (1997). Psychosocial factors predicting employee sickness absence during economic decline. Journal of Applied Psychology 82, 858-872.

Kivimäki, M., Vahtera, J., Koskenvuo, M., Uutela, A. \& Pentti, J. (1998). Response of hostile individuals to stressful change in their working lives: test of a psychosocial vulnerability model. Psychological Medicine 28, 903-913.

Kivimäki, M., Elovainio, M. \& Vahtera, J. (2000a). Workplace bullying and sickness absence in hospital staff. Occupational and Environmental Medicine 57, 656-660.

Kivimäki, M., Vahtera, J., Pentti, J. \& Ferrie, J. E. (2000 b). Factors underlying the effect of organisational downsizing on health of employees: longitudinal cohort study. British Medical Journal 320, 971-975.

Kujala, U. M., Kaprio, J., Sarna, S. \& Koskenvuo, M. (1998). Relationship of leisure-time physical activity and mortality. Journal of the American Medical Association 279, 440-444

Moorman, R. H. (1991). Relationship between organizational justice and organizational citizenship behaviors: do fairness perceptions 
influence employee citizenship? Journal of Applied Psychology 76, 845-855.

Nachmias, C. \& Nachmias, D. (1981). Research Methods in the Social Science. St Martin's Press: New York.

Pattani, S., Constantinovici, N. \& Williams, S. (2001). Who retires early from the NHS because of ill health and what does it cost? A national cross sectional study. British Medical Journal 322 208-209.

Richards, J. C., Hof, A. \& Alvarenga, M. (2000). Serum lipids and their relationships with hostility and angry affect in men. Health Psychology 19, 393-398.

Sarason, I. G., Sarason, B. R., Shearin, E. N. \& Pierce, G. R. (1987). A brief measure of social support: practical and theoretical implications. Journal of Social and Personal Relationships 4, 497-510.

Shapiro, D. L. \& Brett, J. M. (1993). Comparing three processes underlying judgements of procedural justice: a field study of mediation and arbitration. Journal of Personality and Social Psychology 65, 1167-1177.

Stansfeld, S. A., Rael, E. G. S., Head, J., Shipley, M. \& Marmot, M. (1997). Social support and psychiatric sickness absence: a prospective study of British civil servants. Psychological Medicine 27, $35-48$.
Stansfeld, S. A., Fuhrer, R., Shipley, M. J. \& Marmot, M. G. (1999). Work characteristics predict psychiatric morbidity: prospective results from the Whitehall II study. Occupational and Environmental Medicine 56, 302-307.

Tanskanen, A., Tuomilehto, J., Viinamaki, H., Vartiainen, E., Lehtonen, J. \& Puska, P. (2000). Joint heavy use of alcohol, cigarettes and coffee and the risk of suicide. Addiction 95, 1699-1704.

Vahtera, J., Kivimäki, M., Koskenvuo, M. \& Pentti, J. (1997). Hostility and registered sickness absences: a prospective study of municipal employees. Psychological Medicine 27, 693-701.

Vahtera, J., Kivimäki, M., Pentti, J. \& Theorell, T. (2000). Effect of change in the psychosocial work environment on sickness absence: a 7-year follow-up of initially healthy employees. Journal of Epidemiology and Community Health 54, 484-493.

Wall, T. D., Bolden, R. I., Borrill, C. S., Carter, A. J., Golya, D. A., Hardy, G. E., Haynes, C. E., Rick, J. E., Shapiro, D. A. \& West, M. A. (1997). Minor psychiatric disorders in NHS trust staff: occupational and gender differences. British Journal of Psychiatry 171, 519-523.

Williams, S., Michie, S. \& Pattani, S. (1998). Improving the Health of the NHS Workforce: Report of the Partnership on the Health of the NHS Workforce. Nuffield Trust: London. 